\title{
Volume filling factors of the ISM phases in star forming galaxies
}

\section{The role of the disk-halo interaction}

\author{
M. A. de Avillez ${ }^{1}$ and D. Breitschwerdt ${ }^{2,3}$ \\ 1 Department of Mathematics, University of Évora, R. Romão Ramalho 59, 7000 Évora, Portugal \\ e-mail: mavillez@galaxy.lca.uevora.pt \\ 2 Max-Planck-Institut für Extraterrestrische Physik, Giessenbachstraße, Postfach 1312, 85741 Garching, Germany \\ e-mail: breitsch@mpe.mpg.de \\ ${ }^{3}$ Present address: Institut für Astronomie, Universität Wien, Türkenschanzstr. 17, 1180 Wien, Austria \\ e-mail: breitschwerdt@astro.univie.ac.at
}

Received 10 July 2003 / Accepted 23 June 2004

\begin{abstract}
The role of matter circulation between the disk and halo in establishing the volume filling factors of the different ISM phases in the Galactic disk $(|z| \leq 250 \mathrm{pc})$ is investigated, using a modified version of the three-dimensional supernovadriven ISM model of Avillez (2000). We carried out adaptive mesh refinement simulations of the ISM with five supernova rates (in units of the Galactic value), $\sigma / \sigma_{\text {Gal }}=1,2,4,8$ and 16 (corresponding to starburst conditions) using three finer level resolutions of $2.5,1.25$ and $0.625 \mathrm{pc}$, allowing us to understand how resolution would affect the volumes of gas phases in pressure equilibrium. We find that the volume filling factors of the different ISM phases depend sensitively on the existence of a duty cycle between the disk and halo acting as a pressure release valve for the hot $\left(T>10^{5.5} \mathrm{~K}\right)$ phase in the disk. The amount of cold gas (defined as the gas with $T<10^{3} \mathrm{~K}$ ) picked up in the simulations varies from a value of $19 \%$ for $\sigma / \sigma_{\text {Gal }}=1$ to $\sim 5 \%$ for $\sigma / \sigma_{\mathrm{Gal}}=4$ and $\leq 1 \%$ for higher $\mathrm{SN}$ rates. Background heating prevents the cold gas from immediate collapse and thus ensures the stability of the cold gas phase. The mean occupation fraction of the hot phase varies from about $17 \%$ for the Galactic SN rate to $\sim 28 \%$, for $\sigma / \sigma_{\text {Gal }}=4$, and to $44 \%$ for $\sigma / \sigma_{\text {Gal }}=16$. Overall the filling factor of the hot gas does not increase much as we move towards higher SN rates, following a power law of $\left\langle f_{v \text {, hot }}\right\rangle \propto\left(\sigma / \sigma_{\text {Gal }}\right)^{0.363}$. Such a modest dependence on the $\mathrm{SN}$ rate is a consequence of the evacuation of the hot phase into the halo through the duty cycle. This leads to volume filling factors of the hot phase considerably smaller than those predicted in the three-phase model of McKee \& Ostriker (1977) even in the absence of magnetic fields.
\end{abstract}

Key words. galaxies: ISM - galaxies: kinematics and dynamics - ISM: structure

\section{Introduction}

In their seminal paper of a three-phase model regulated by supernova explosions in an inhomogeneous medium, McKee \& Ostriker (1977) predicted a volume filling factor of the hot intercloud medium (HIM) of $f_{v \text {, hot }}=0.7-0.8$. However, observations point to a value of $\sim 0.5$ (e.g., Dettmar 1992) or even lower when external galaxies are taken into account (e.g., Brinks \& Bajaja 1986). A way out has been suggested by Norman \& Ikeuchi (1989) by the so-called chimney model, in which hot gas can escape into the halo through tunnels excavated by clustered supernova $(\mathrm{SN})$ explosions. Indeed X-ray observations of several nearby edge-on galaxies have revealed extended, galaxy-sized halos (e.g., Wang et al. 2001). The transport of gas into the halo is, however, still controversial, and arguments that superbubble (SB) break-out may be inhibited by a large-scale disk parallel magnetic field have been put forward by several authors (e.g., Mineshige et al. 1993).
It is true that magnetic tension forces can in principle considerably decelerate an upward flow, so that it will eventually stall before break-out. Tomisaka (1998) has performed 3D MHD simulations of expanding superbubbles including radiative cooling. He finds that bubble confinement only occurs when the energy injection rate is below a critical value of $\sim 10^{37} \mathrm{erg} \mathrm{s}^{-1}$ (see also MacLow \& McCray 1988) and/or the scale height of the field is infinite. Coupling the field distribution to the vertical gas density distribution, as seems more realistic for frozen-in field lines, e.g. $B \propto \rho^{1 / 2}$, break-out will occur even if the midplane field strength is as high as $5 \mu \mathrm{G}$.

Attempts to determine the occupation fraction of the different phases, and in particular of the hot gas, by means of modelling the effects of SNe and SBs in the ISM, have been carried out by several authors (e.g., Ferrière 1995, 1998; Korpi et al. 1999). However, these models do not include the circulation of gas between the disk and the full halo, thus being unable to resolve the high- $z$ region; neither do they take into account the 
mixing between the different phases. Therefore, an estimate of the volume filling factors may be misleading.

Large scale simulations of the ISM in 2D (Rosen \& Bregman 1995) and in 3D (Avillez 2000; Avillez \& Berry 2001) show the importance of the disk-halo interaction in the dynamics and evolution of the ISM in disk galaxies, which is intimately related to the vertical structure of the thick gas disk and to the rate of occurrence of supernovae per unit area in the Galactic disk. A major consequence of this interaction is the establishment of a duty cycle between the disk and halo by hot gas breaking through the thick gas disk, either in a violent way through chimneys or in a secular fashion through the buoyant rising of hot gas (Avillez 2000; Avillez \& Mac Low 2001), into the upper parts of the thick gas disk (the disk-halo interface). From here it escapes into the halo setting up a Galactic fountain (some of this gas may escape from the Galaxy as a wind, cf. Breitschwerdt et al. 1993) whose major fraction returns to the disk as cold gas (after condensing into clouds), interacting with the thin gas disk.

In this paper we investigate how the volume filling factors of the different ISM phases in the Galactic disk (which we consider hereafter to be the region with $-250 \leq z \leq 250 \mathrm{pc}$ ) evolve with the local Galactic SN rate (varying from the present value to a factor of 16 times higher) over a sufficiently long time scale in order to set up a dynamical equilibrium condition. We show that matter circulation including the Galactic halo is an important ingredient in explaining the volume filling factors of the stable phases in the ISM, in particular a much lower value for the hot intercloud medium (HIM), even without magnetic fields, is obtained than advocated by McKee \& Ostriker (1977). The present study makes use of a modified version of the supernova-driven ISM model of Avillez (2000) and carries out simulations on grids of kpc-scale size with a spatial resolution as fine as $1.25 \mathrm{pc}$ and $0.625 \mathrm{pc}$, respectively, in order to study the ISM in the disk and halo of star forming galaxies within a wide range of different supernova rates. The simulation time scales are of the order of $400 \mathrm{Myr}$ so that the disk-halo-disk cycle can be fully established, thus allowing the system to completely lose its memory of the initial conditions.

In Sect. 2 we review the three-dimensional SN-driven ISM model used in the present work and the numerical schemes applied to it, as well as the set-up of the current study. In Sect. 3, we describe the global evolution of the ISM as seen in these runs. Section 4 discusses the results and their implications. Finally Sect. 5 presents a summary of the main results and final remarks.

\section{SN-Driven ISM modelling}

\subsection{Numerics}

In the current work we report on large-scale simulations of the ISM including the disk and Galactic halo using a modified version of the SN driven ISM model of Avillez (2000) coupled to a three-dimensional HD code using adaptive mesh refinement (AMR) in a block-based structure, that relies on virtual topologies of CPUs created through Message Passing Interface
(MPI) calls. This topology is associated to the computational domain that is divided into blocks, each having $N=n_{x} \times n_{y} \times n_{z}$ cells. When a refinement is required, a block is split into eight new blocks (children) of $N$ cells. This process is repeated until the finest level of resolution is reached. All the information relative to the tree structure is preserved in the virtual topology, being only necessary to query the different CPUs to learn their location in this topology, and therefore, the location of their neighbors, children and parents. Each grid block is refined periodically in regions where steep pressure gradients appear. The local increase of the number of cells corresponds to an increase in linear resolution by a factor of two. The adaptive mesh refinement scheme is based on Berger \& Colella (1989), but the grid generation procedure follows that described in Bell et al. (1994). The gas dynamics part of the code uses the piecewise-parabolic method of Colella \& Woodward (1984), a third-order scheme based on a Godunov method implemented in a dimensionally-split manner (Strang 1968) that relies on solutions of the Riemann problem in each zone rather than on artificial viscosity to follow shocks.

\subsection{Model}

\subsubsection{Setup of basic processes}

The present model introduces substantial improvements to that of Avillez (2000), namely: (i) inclusion of background heating due to starlight varying with $z$ (Wolfire et al. 1995) and kept constant in the directions parallel to the Galactic plane $(z=0)$ where it is chosen to initially balance radiative cooling at $9000 \mathrm{~K}$. With the inclusion of background heating the gas at $T<10^{4} \mathrm{~K}$ becomes thermally bistable; (ii) use of a tabulated cooling function taken from Dalgarno \& McCray (1972) with an ionization fraction of 0.1 at temperatures below $10^{4} \mathrm{~K}$ and a temperature cutoff at $10 \mathrm{~K}$; (iii) inclusion of SNe type Ia with a scale height of 325 pc (Freeman 1987); (iv) OB stars form in regions with a density and temperature thresholds of $10 \mathrm{~cm}^{-3}$ and $100 \mathrm{~K}$, respectively, and their number, masses and main sequence life times $\tau_{\mathrm{MS}}$ are determined from the initial mass function. Roughly sixty percent of the OB stars explode within the cluster, while the remaining (composed of the lowest mass stars whose location is determined kinematically by attributing to each star a random direction and a velocity) explode in the field with a scale height of $90 \mathrm{pc}$. The associations form in a layer with a scale height of $46 \mathrm{pc}$. The time interval between the explosions of all OB stars is determined by their $\tau_{\mathrm{MS}}$ for the given $\mathrm{SN}$ rate.

As in the Avillez (2000), the present model includes the fixed vertical gravitational field provided by the stars in the disk and the interstellar gas is initially setup with a density stratification distribution that includes the cold, cool, warm, ionized and hot gases as described in Ferrière (1998). This ignores, by the way, curvature terms in the perpendicular $r-z$-plane, that may become relevant at some distance from the plane and induce radial motions. On the other hand, this effect will be small as long as the flow remains supersonic, since the gas has virtually no time to establish lateral pressure equilibrium. 


\subsubsection{Further processes}

Owing to the observational complexity of the ISM and the nonlinearity of the dominant processes occurring there, we have set up a model with the most important ingredients, neglecting other processes that may be relevant too, like differential rotation in the case of extended horizontal gas flows, selfgravity in case of Jeans unstable clouds, the gravitational field of a dark matter halo component, thermal conduction, and, most importantly, magnetic fields. The latter is discussed in Avillez \& Breitschwerdt (2004a,b). Self-gravity is neglected, since with the cooling function we are using, we are not able to treat the formation of molecular clouds (also dust cooling is not included). In this respect our calculations are incomplete. However, it is known that the molecular phase decouples due to gravitational instability, and hence is not even in rough pressure equilibrium with the other ISM phases.

The reason for attacking the problem in this way, in contrast to other authors who try to implement as many processes as possible with computing power being the major limitation, is the following. We believe that in order to gain insight into the nonlinear physics of a complex system like the ISM, driven by temporally and spatially variable energy input, we should first try to understand the interplay and effects of the most dominant processes. Therefore we do not strive for completeness here, but for a better understanding of the ISM gas dynamics. As a brief comparison with the observations shows, and, as will be shown in more detail in the second paper, the quantitative values that we obtain for the volume filling factors of the ISM gas are already in remarkably good agreement with the data. Below we discuss in some detail the relevance of processes that are known to be important in the ISM, but have been neglected in the present simulations.

- Thermal conduction: the importance of thermal conduction in the ISM has been the subject of debate for at least 25 years. There is some agreement that Spitzer conductivity does certainly overestimate the heat flux by a large amount, since the inclusion of an even weak magnetic field reduces the mean free path of the electrons to a gyroradius, which is orders of magnitude below any astrophysical length scale. Saturated conduction (e.g. Cowie \& McKee 1977 ) is an improvement, when steep temperature gradients occur. In this case the heat flux will be independent of the temperature gradient. But the flux would be reduced by $\cos \theta$, where $\theta$ is the angle between the local magnetic field direction and the temperature gradient. It has been argued recently in the context of cooling flows in clusters of galaxies (e.g. Narayan \& Medvedev 2001), that turbulence may boost the conduction rate and thus overcome the suppression by a magnetic field. However, as has been pointed out by Malyshkin \& Kulsrud (2001), there are two effects that inhibit heat flow even in the case of heat conduction in stochastic magnetic fields: (1) the electrons have to spiral along the lines of force, which are highly tangled, and therefore have to drift a long way, encountering on average smaller temperature gradients; (2) the electrons may become temporarily trapped on their journey by magnetic mirrors, until released by decreasing their pitch angle sufficiently by collisions.

We have not included heat conduction in the present paper, since as a second order process it should be slower than hydrodynamic mixing. As is shown in this paper, supernova induced turbulence is quite strong in the ISM, and therefore we think that turbulent diffusion, induced by turbulent motions, will be the more efficient mixing process.

- Coriolis forces: the present set-up ignores Galactic rotation, which can give rise to radial motions. A measure of the importance of Coriolis forces with respect to inertial forces is the Rossby number $\epsilon_{\mathrm{R}}=v /\left(\Omega_{0} L\right) \ll 1$, where $L \sim 1 \mathrm{kpc}$ is the maximum size of our grid. As far as the hot medium is concerned the rms velocity component is of the order of $100 \mathrm{~km} \mathrm{~s}^{-1}$ and hence $\epsilon_{\mathrm{R}} \sim 4$, for $\Omega_{0}=8.4 \times 10^{-16} \mathrm{~s}^{-1}$. Moreover, the hot gas breaking out of the disk following the density gradient has a dominant $z$-component, for which the Coriolis term vanishes.

- Dark matter halo: up to a distance of $\sim 10 \mathrm{kpc}$ the amount of dark matter pulling the gas down is small compared to the disk potential. To see this, one can look at the gravitational potential, for which in case of the Milky Way one can use for the bulge and disk component the mass model of Miyamoto \& Nagai (1975) plus a spherical dark matter halo component as suggested by Innanen (1973). Comparing the gravitational acceleration terms near the solar circle, $g_{\text {disk }}$ and $g_{\text {halo }}$ of the bulge plus disk and the dark matter halo, respectively, for a fluid element at vertical distances $z=5$ and $z=10 \mathrm{kpc}$, one obtains $\left[g_{\text {disk }}(z=5)=1.19 \times 10^{-8}\right.$, $\left.g_{\text {halo }}(z=5)=1.23 \times 10^{-9}\right]$ and $\left[g_{\text {disk }}(z=10)=8.72 \times 10^{-9}\right.$, $\left.g_{\text {halo }}(z=10)=1.96 \times 10^{-9}\right]$, in CGS units respectively. Only at $z \approx 35 \mathrm{kpc}$ do the two terms become comparable, with the halo component dominating at larger heights. Thus neglecting the dark matter component in the simulations, where $z \leq 10 \mathrm{kpc}$, is fully justified for spiral galaxies.

\subsection{Simulations}

The simulations use a computational domain that contains a section of the Galaxy with an area of $1 \mathrm{kpc}^{2}$ and a vertical extension from -10 to $10 \mathrm{kpc}$. The innermost edge lies $8.5 \mathrm{kpc}$ from the Galactic centre, coinciding with the solar circle. The grid resolution is $10 \mathrm{pc}$ except in the layer between -500 and $500 \mathrm{pc}$, where two, three and four levels of AMR are used, yielding a finest level resolution of $2.5,1.25$ and $0.625 \mathrm{pc}$, respectively. For the 2.5 and $1.25 \mathrm{pc}$ resolutions five runs using $\sigma / \sigma_{\mathrm{Gal}}=1,2,4,8$ and 16 were carried out, while the $0.625 \mathrm{pc}$ runs used $\sigma / \sigma_{\text {Gal }}=1,2$ and 4 . At the cap surfaces $z=-10 \mathrm{kpc}$ and $z=10 \mathrm{kpc}$ outflow boundary conditions were imposed and periodic ones along the four vertical boundary surfaces. The simulations were evolved for 400 Myr. Such a large grid size and long evolution time scale is needed to follow the time-dependent evolution of the ISM allowing several generations of young massive stars to occur within the computational domain. The highest resolution simulations are used to answer the crucial question: does each refinement of the grid lead to a qualitatively and quantitatively different picture of the ISM or 
does the distribution of gas over the various phases converge to values depending mainly on the $\mathrm{SN}$ rate and not on grid resolution?

\section{Results}

\subsection{Global evolution of the ISM}

The simulations start with a set of hydrodynamic and thermodynamic variables $\rho, P_{\text {gas }}$ and $T$ taken from observations. However, the initially stratified distribution does not hold for long as a result of the lack of equilibrium between gravity and (thermal, kinetic and turbulent) pressure during the "switchon" phase of SN activity. As a consequence the gas distributions in the upper $(z=10 \mathrm{kpc})$ and bottom $(z=-10 \mathrm{kpc})$ parts of the grid collapse into the midplane, leaving low density material behind. When enough supernovae have gone off in the disk building up the required pressure support most of the collapsed gas expands supersonically through the grid filling all the computational domain. A continuous flow between the disk and halo is then set up, where the upward and downward flowing gas come into some sort of dynamical equilibrium.

The gas in the disk also reaches equilibrium, although on a different time scale, mainly determined by the input of energy into the ISM by SNe, diffuse heating and the energy lost by adiabatic (due to expansion of SNRs, SBs and escaping disk gas) and radiative cooling. The disk equilibrium is related to the time scale that pressure needs to build up there as a result of SN explosions, while on the global halo scale such an equilibrium is only possible after the full establishment of the duty cycle of the warm and hot gas and its circulation between the disk and the halo, which takes several hundred Myr. An upper limit for this can be estimated by calculating the flow time, $\tau_{\mathrm{f}}$, that the gas needs to travel to the critical point of the flow in a steady-state (see Kahn 1981). This is the characteristic distance from which information in a thermally driven flow can be communicated back to the sources. Then $\tau_{\mathrm{f}} \sim r_{\mathrm{c}} / c_{\mathrm{s}}$, where $r_{\mathrm{c}}$ and $c_{\mathrm{s}}$ are the location of the critical point and the speed of sound, respectively. For spherical geometry, the critical point can be simply obtained from the steady state fluid equations, $r_{\mathrm{c}} \sim G M_{\mathrm{gal}} /\left(2 c_{\mathrm{s}}^{2}\right)$, which yields with a Milky Way mass of $M_{\text {gal }} \approx 4 \times 10^{11} M_{\odot}$ a distance $r_{\mathrm{c}} \approx 31.3 \mathrm{kpc}$ for an isothermal gas at $T=2 \times 10^{6} \mathrm{~K}$ (corresponding to a sound speed of $1.67 \times 10^{7} \mathrm{~cm} \mathrm{~s}^{-1}$ ), and thus $\tau_{\mathrm{f}} \sim 180 \mathrm{Myr}$ as an upper limit for the flow time. For comparison, the radiative cooling time of the gas at a typical density of $n=2 \times 10^{-3} \mathrm{~cm}^{-3}$ is roughly $\tau_{\mathrm{c}} \sim 3 k_{\mathrm{B}} T /(n \Lambda) \approx 155$ Myr for a standard collisional ionization equilibrium cooling function $\Lambda=8.5 \times 10^{-23} \mathrm{erg} \mathrm{cm}^{3} \mathrm{~s}^{-1}$ of gas with cosmic abundances. This value is apparently of the same order as the flow time, ensuring that the flow will not only cool by adiabatic expansion, but also radiatively, thus giving rise to the fountain return flow, which is the part of the outflow that loses pressure support from below and therefore cannot escape. Note that $r_{\mathrm{c}}$ is the maximum extension of the fountain, more than an order of magnitude larger, than in simulations with grids with a vertical extension of $1 \mathrm{kpc}$ above and below the midplane. Those calculations definitely miss an important component of the galactic gas dynamics.
In the disk, on the other hand, the cooling time scale is much shorter than in the halo, because of the higher ISM densities there. We thus find from our simulations that typical time scales of pressure fluctuations about a mean value of $P / k_{\mathrm{B}} \sim 2600 \mathrm{~cm}^{-3} \mathrm{~K}$ are of the order of $30 \mathrm{Myr}$, which is by the way also a typical time scale for superbubble evolution.

However, it should be emphasized, that since disk and halo are coupled dynamically not only by the escape of hot gas, but also by the fountain return flow striking the disk, the disk equilibrium will suffer secular variations on time scales of the order of 100-200 Myr.

Cuts through the 3D data cube at $z=0 \mathrm{pc}$ and taken at 400 Myr presented in Fig. 1 show the density (left column), $P / k$ (middle column) and temperature (right column) distributions in the Galactic plane at $400 \mathrm{Myr}$ of evolution, well after the system has reached dynamical equilibrium. The supernova rates in units of the Galactic value are: 1,2, and 4, and the resolution of the finest AMR level is $1.25 \mathrm{pc}$. These images show the structure and morphology of the different ISM phases defined here as: cold $\left(T<10^{3} \mathrm{~K}\right)$, cool $\left(10^{3}<T<10^{4} \mathrm{~K}\right)$, warm $\left(10^{4}<T<10^{5.5} \mathrm{~K}\right)$ and hot $\left(T>10^{5.5} \mathrm{~K}\right)$. The morphology of the ISM and the volume filling factors of the various phases obviously vary with SN rate. Not surprisingly, the amount of cold gas reduces with increasing SN rate, while the amount of the warm and hot gas increases. The images also show the expansion of SNRs and the interaction of shock waves, which can be best observed in the pressure maps where the shocks are represented by high pressure and thin structures in red. This is not seen in the density or temperature maps. A comparison between these maps shows that most of these shocks are located at the interfaces between hot and intermediate temperature gas, i.e., $10^{4}<T<10^{5.5} \mathrm{~K}$.

Inspection of the pressure distribution shows that the most abundant colour is yellow-green, implying an average $P / k \sim$ $2600 \mathrm{~K} \mathrm{~cm}^{-3}$ (see also Fig. 2, upper right panel) for $\sigma=\sigma_{\mathrm{Gal}}$, and about 10000 for $\sigma=4 \sigma_{\mathrm{Gal}}$. This is in agreement with the observational fact that the cool, warm and hot phase are in rough pressure equilibrium. This should however not be confused with a local equilibrium. On the contrary, severe deviations from the equilibrium values can occur locally due to $\mathrm{SN}$ activity and thermal instabilities (see the red an blue regions in Fig. 1, middle column).

For the Galactic SN rate, once the system reaches its equilibrium configuration, a thin disk of cold gas forms in the Galactic plane, and, above and below, a thick inhomogeneous gas disk forms (this vertical structure of the disk is similar to that found in Avillez 2000). The code does not explicitly follow ionization states, but we can trace cool gas with a temperature $T \leq 10^{4} \mathrm{~K}$ and a scale height of $180 \mathrm{pc}$, and warm gas with $10^{4} \leq T \leq 10^{5} \mathrm{~K}$ and a scale height of $1 \mathrm{kpc}$. These distributions reproduce those described in Dickey \& Lockman (1990) and Reynolds (1987), respectively. The thick gas disk is punctured by chimneys that result from superbubbles occurring on either side of the midplane at a height greater than $100 \mathrm{pc}$. As they grow, they elongate along the $z$-direction, owing to the local density stratification of the ISM. Chimneys in the simulation typically have widths of approximately $150-200 \mathrm{pc}$. They inject high temperature gas directly from the Galactic disk into 

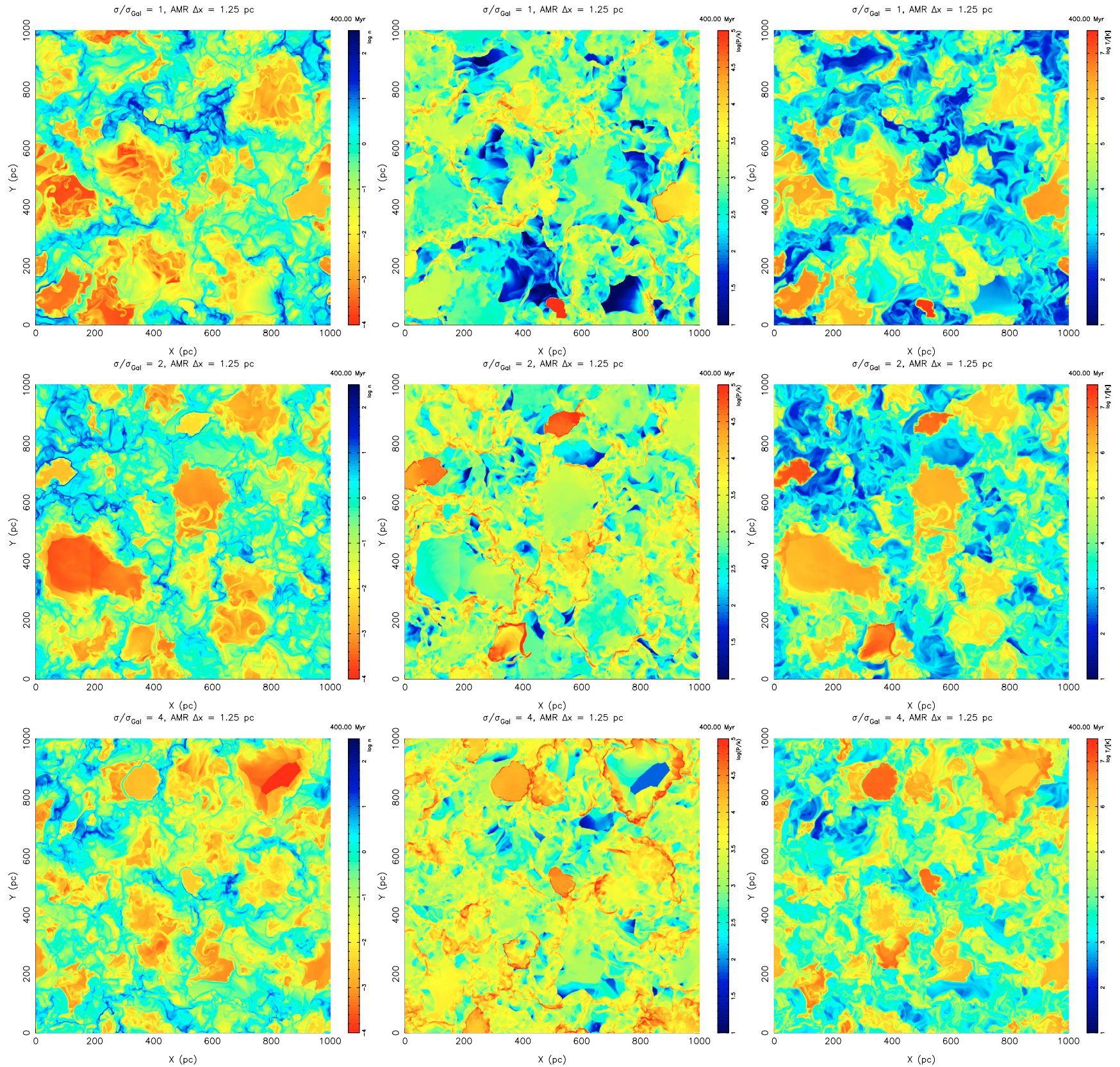

Fig. 1. Two dimensional cuts, through the $3 \mathrm{D}$ data cube, showing the $\rho / \rho_{0}$ (first column), $P / k$ (middle column) and $T$ (right column) distribution in the Galactic plane for $\sigma / \sigma_{\mathrm{Gal}}=1$ (first row), 2 (second row) and 4 (bottom row).

the halo, breaking through the cool and warm layers that compose the thick disk. This hot gas then contributes to the galactic fountain. Thus, the upper parts of the thick warm disk form the disk-halo interface, where a large scale fountain is set up by hot ionized gas escaping in a turbulent convective flow.

For higher $\mathrm{SN}$ rates a similar $z$-structure of a thick gas disk is seen, but with a higher vertical extension. This is mainly due to the rate of $\mathrm{SNe}$ in the field rather than to the clustered $\mathrm{SNe}$, which drive superbubbles breaking through the thick gas disk and injecting their matter directly into the halo. The evolution of the vertical structure of star-forming galaxies with different $\mathrm{SN}$ rates will be discussed in more detail in a forthcoming paper.

\subsection{Probability distribution functions}

A major consequence of the set-up of the disk-halo cycle by SN activity is that the system loses any recollection of its initial conditions as most of the disk gas has already travelled into the halo and came back to the disk. Thus, the effects of initial conditions are not present in the temperature probability distribution functions (PDFs) of the system for different SN rates.

Figure 2 compares volume weighted histograms of the temperature over the periods of 0-50 Myr (red) and 350-400 Myr (black) calculated using 50 snapshots taken at time intervals of $1 \mathrm{Myr}$ for the runs with $\sigma / \sigma_{\mathrm{Gal}}=1,4$, and 8 and a finest AMR resolution of $1.25 \mathrm{pc}$. These PDFs indicate that for low $\sigma$, 

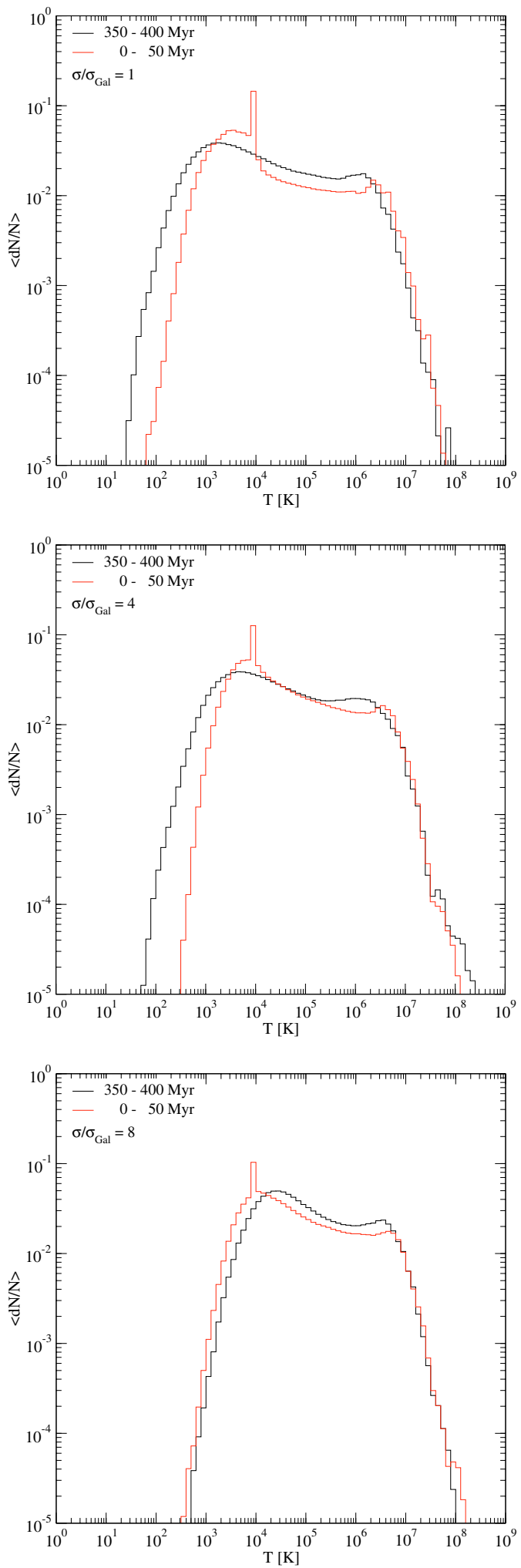

Fig. 2. Averaged volume-weighted temperature PDFs over the periods of 0-50 Myr (red) and 350-400 Myr (black) calculated using 51 snapshots taken at time intervals of $1 \mathrm{Myr}$. The supernova rates used in these models are: $\sigma / \sigma_{\mathrm{Gal}}=1$ (top panel), 4 (middle panel), and 8 (bottom panel). The resolution of the finest AMR level is $1.25 \mathrm{pc}$.

the temperature peak is at about $2000 \mathrm{~K}$, making the cold/warm HI gas the most abundant gas phase, consistent with the density PDFs (Fig. 3) and also with observations. This peak
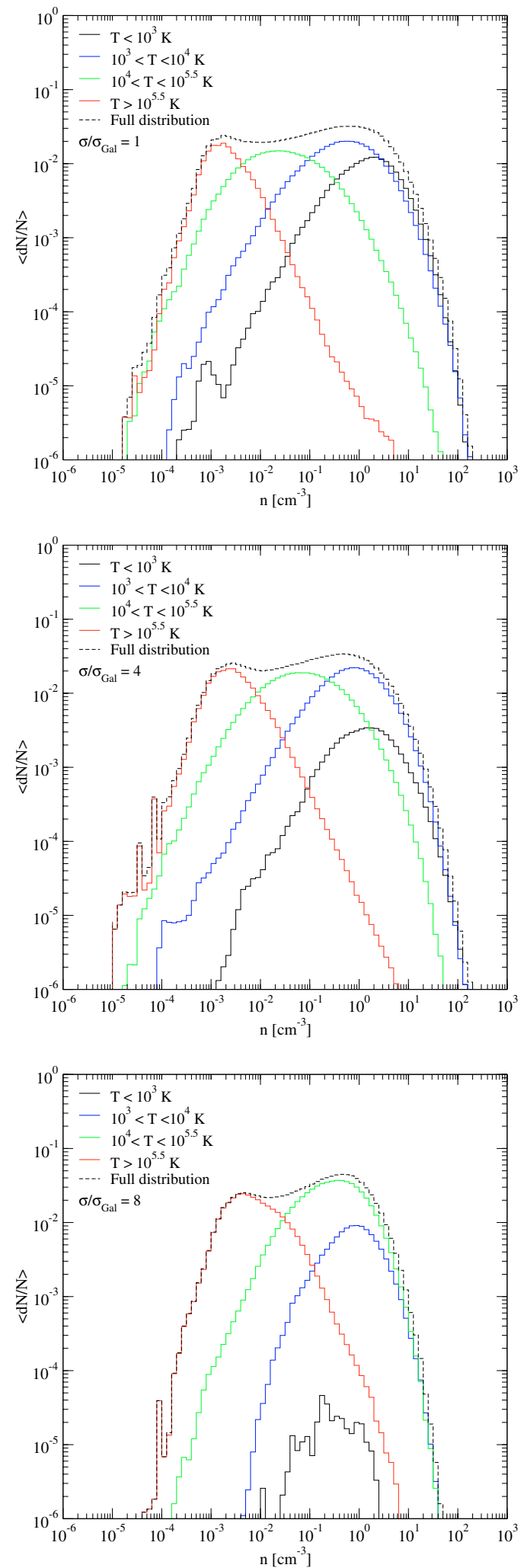

Fig. 3. Averaged volume-weighted density PDFs over the period 350-400 Myr calculated using 51 snapshots taken at time intervals of $1 \mathrm{Myr}$ for the SN rates shown in Fig. 2. The finest level AMR resolution is $1.25 \mathrm{pc}$.

is shifted towards $T \approx 40000 \mathrm{~K}$, for $\sigma=16 \sigma_{\mathrm{Gal}}$, making the warm phase the most important one. At the same time the relative importance of the hot phase increases as well, and one is 
moving towards a bimodal distribution. It can be directly seen from Fig. 2 that a simulation time of only $50 \mathrm{Myr}$ is not sufficient to obtain this result, mainly because the effect of upwards transport is to establish a duty cycle, which takes of the order of a few hundred Myrs. In any of the cases shown in the figures the averaged PDFs for the initial 50 Myr have two pronounced peaks, one around $8000 \mathrm{~K}$ and the other around $5 \times 10^{6} \mathrm{~K}$. Note that with the increase of SN rate the PDFs of the first $50 \mathrm{Myr}$ suffer large variations, indicating that the loss of recollection of the initial conditions will occur earlier for the highest $\mathrm{SN}$ rates, suggesting that in a time-asymptotic sense the $\mathrm{SN}$ rate is the controlling parameter. The corresponding averaged volume-weighted PDFs of the density and pressure distribution in the Galactic disk over the period 350-400 Myr are shown in Figs. 3 and 4. It can be seen that there are three distinct peaks in the density PDFs corresponding to the three most abundant regimes (cool, warm and hot). The cold regime has a peak that decreases steeply with increase of SN rate. These peaks have similar pressure ranges. The pressure PDFs show that the range of the total pressure (dashed lines) decreases slightly with increase of the SN rate. For $\sigma=\sigma_{\mathrm{Gal}}$ and $\langle\mathrm{d} N / N\rangle=10^{-2}$, total pressure spans three orders of magnitude from $10^{-14}$ to $10^{-11}$, while for higher $\mathrm{SN}$ rates of 8 and 16 times the Galactic value the total pressure spans three and two orders of magnitude, respectively. This is indicative of the large variation in the pressure distribution between the different temperature regimes, and suggests that there are no real phases, i.e. co-existing thermodynamic regimes with different density and temperature but in pressure equilibrium.

\subsection{Volume filling factors}

At $\sigma=\sigma_{\text {Gal }}$, the hot phase has a volume filling factor $f_{v \text {, hot }} \sim 0.17$, comparable to that of the cold gas, and the warm/cool phase is dominating. Increasing to 4, 8 and 16 times $\sigma_{\text {Gal }}$ pushes the warm gas to take over in volume, while there is a slight increase of the occupation fraction of the hot gas. It is interesting to note that the peak of the warm gas still surpasses that of the hot gas in magnitude even for a factor of 16 (see Fig. 6). This must be due to the fact that the reservoir of cold gas is used up by increased Lyman continuum photon absorption and shock heating, whereas the hot gas escapes into the halo. Between $\sigma=4$ and $16 \sigma_{\mathrm{Gal}}$, the cold/cool phase is reduced substantially and eventually to insignificance. This must have direct consequences for the formation of molecular clouds and thus for continuous star formation. It seems plausible that this leads eventually to self-regulation.

Figure 5 shows the history of of the volume filling factors of the different phases in the simulated disk for $\sigma / \sigma_{\mathrm{Gal}}=1$, 4 , and 8, while Table 1 and Fig. 6 show the variation with supernova rate of the time averaged volume filling factors of the different phases, over the period 300-400 Myr calculated using 101 snapshots with a time interval of 1 Myr.

The distribution of the occupation fraction of the different phases comes to an equilibrium at about $100 \mathrm{Myr}$ for $\sigma / \sigma_{\text {Gal }}>1$, while for the Galactic SN rate it occurs somewhat later, i.e. at about 200 Myr. This is a result of the set-up
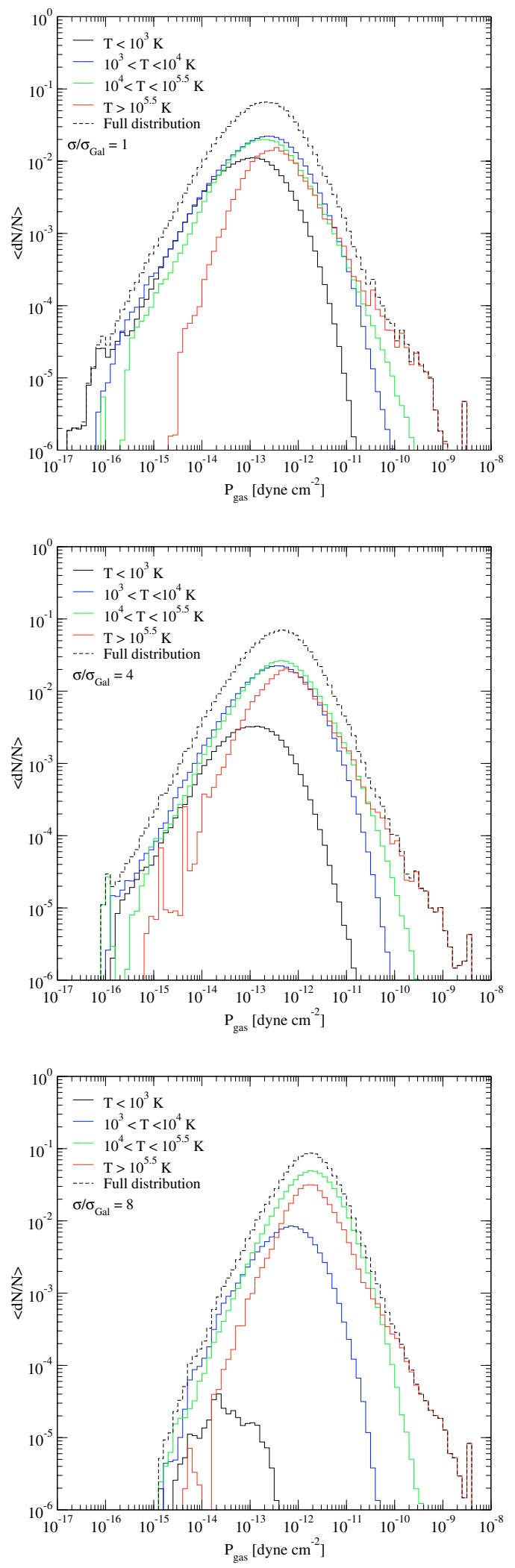

Fig. 4. Averaged volume-weighted pressure PDFs over the period 350-400 Myr calculated using 51 snapshots taken at time intervals of 1 Myr for the SN rates shown in Fig. 2. The finest level AMR resolution is $1.25 \mathrm{pc}$.

of the disk-halo cycle, which for the Galactic SN rate takes about 200 Myr to establish, while for higher SN rates it takes 

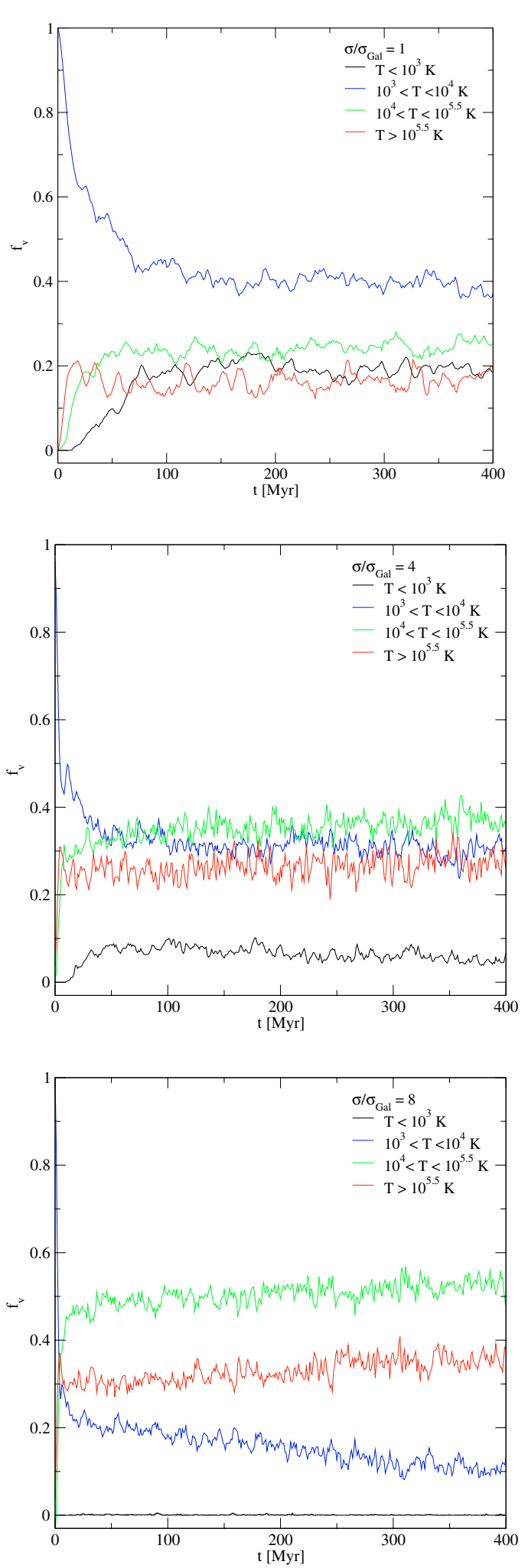

Fig. 5. Time evolution of the volume filling factors of the cold $(T \leq$ $\left.10^{3} \mathrm{~K}\right)$, cool $\left(10^{3}<T \leq 10^{4} \mathrm{~K}\right)$, warm $\left(10^{4}<T \leq 10^{5.5} \mathrm{~K}\right)$, and hot $\left(T>10^{5.5} \mathrm{~K}\right)$ phases for $\sigma / \sigma_{\mathrm{Gal}}=1$ (top panel), 4 (middle panel), and 8 (bottom panel). The finest AMR level resolution is $1.25 \mathrm{pc}$. Note that the line for the cold gas is slightly over $f_{v}=0$, for $\sigma / \sigma_{\mathrm{Gal}}=8$.

less time in evacuating the hot gas from the disk. This is presumably due to an over-pressure with respect to the ambient

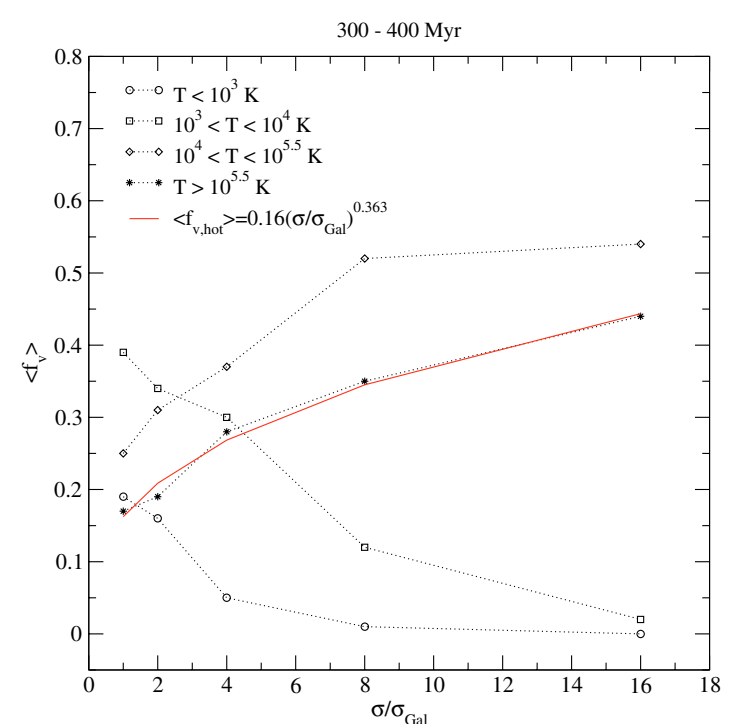

Fig. 6. Variation of the average volume filling factor of $T<10^{3}$ (circles), $10^{3}<T<10^{4}$ (squares), $10^{4}<T<10^{5.5}$ (diamonds) and $T>10^{5.5} \mathrm{~K}$ (stars) phases in the simulated disk (for $|z|<250 \mathrm{pc}$ ). The filling factors were averaged using 101 snapshots (of the 1.25 resolution runs) separated by 1 Myr between 300 and 400 Myr of the disk evolution. Red represents a power law fit to $\left\langle f_{v \text {, hot }}\right\rangle$ with the power of 0.363 .

Table 1. Average volume filling factors of the different ISM phases for variable $\mathrm{SN}$ rate. The average was calculated using 101 snapshots (of the 1.25 resolution runs) between 300 and 400 Myr of system evolution with a time interval of $1 \mathrm{Myr}$.

\begin{tabular}{ccccc}
\hline \hline$\sigma^{a}$ & $\left\langle f_{v, \text { cold }}\right\rangle^{b}$ & $\left\langle f_{v, \text { cool }}\right\rangle^{c}$ & $\left\langle f_{v, \text { warm }}\right\rangle^{d}$ & $\left\langle f_{v, \text { hot }}\right\rangle^{e}$ \\
\hline 1 & 0.19 & 0.39 & 0.25 & 0.17 \\
2 & 0.16 & 0.34 & 0.31 & 0.19 \\
4 & 0.05 & 0.30 & 0.37 & 0.28 \\
8 & 0.01 & 0.12 & 0.52 & 0.35 \\
16 & 0.0 & 0.02 & 0.54 & 0.44 \\
\hline
\end{tabular}

${ }^{a} \mathrm{SN}$ rate in units of the Galactic $\mathrm{SN}$ rate.

${ }^{b} T<10^{3} \mathrm{~K}$.

${ }^{c} 10^{3}<T \leq 10^{4} \mathrm{~K}$.

${ }^{d} 10^{4}<T \leq 10^{5.5} \mathrm{~K}$.

${ }^{e} T>10^{5.5} \mathrm{~K}$.

medium and a higher sound speed of the injected hot gas. But in any case, the timescale for the cycle to be completed, even with higher SN rates, is always larger than $100 \mathrm{Myr}$, thus ruling out simulations with lower evolution times.

Most remarkably, for $\sigma / \sigma_{\mathrm{Gal}}=1$, the hot $\left(T>10^{5.5} \mathrm{~K}\right)$ gas has a moderately low volume filling factor (it fluctuates around 0.17 ) in agreement with observations ( 20\%) even in the absence of magnetic fields, and is mainly distributed in an interconnected tunnel network, and in some cases it is even confined to isolated bubbles as seen in Fig. 1. With the increase of the supernova rate to four and eight times the galactic rate the occupation volume of the hot gas increases to about $28 \%$ and $35 \%$, respectively, after 400 Myr of disk evolution, which 
is still below the predictions of McKee \& Ostriker (1977). Even for $\sigma / \sigma_{\mathrm{Gal}}=16$, corresponding already to starburst conditions, the volume fraction of the hot gas increases only to $44 \%$ after $400 \mathrm{Myr}$ of evolution. Such a behaviour can be approximated analytically by a power law fit (Fig. 6)

$\left\langle f_{v \text {,hot }}\right\rangle=0.16\left(\frac{\sigma}{\sigma_{\mathrm{Gal}}}\right)^{0.363}$,

with a a rms percent error of 0.01 .

The warm gas becomes the dominant phase for $\sigma / \sigma_{\mathrm{Gal}}>2$. Between $\sigma / \sigma_{\mathrm{Gal}}=8$ and 16 the occupation volume of this phase has a very small increase from 0.52 to 0.54 , as a result of the conversion of part of the cool gas into this phase. The decrease in the cool gas is a result of its conversion into the warm and hot phases. The growth of the hot phase in time is a mild one even with the large increase in the supernova rate. This is a consequence of the circulation of matter from the disk into the halo which acts as a pressure release valve for the disk gas. As the hot gas is vented into the halo, there is enough space for the $10^{4}<T<10^{5.5} \mathrm{~K}$ gas to be redistributed in the disk. Furthermore, as there is recycling, low temperature gas continues to flow into the disk, thus contributing to the maintenance of the intermediate phases.

For $\sigma / \sigma_{\text {Gal }}=2$ there seems to be a small difference in the volume occupation of the cool (around 34\%) and warm (around $31 \%$ ) phases, while the occupation fraction of the cold gas differs from these by at most $15 \%$ of the total volume. It is interesting to note that the volume occupation of the cool gas decreases some $5 \%$ with the doubling of the $\mathrm{SN}$ rate for $\sigma / \sigma_{\text {Gal }} \leq 4$. On the other hand, there is a reduction of almost $70 \%$ of the filling factor of the cold gas when the supernova rate is increased to $\sigma / \sigma_{\mathrm{Gal}}=4$. This fraction reduces to less than $1 \%$ at $\sigma / \sigma_{\mathrm{Gal}}=8$, disappearing completely for higher $\mathrm{SN}$ rates.

\subsection{Resolution effects}

Our simulations show how crucial spatial resolution is in order to capture small scale structures and promote the mixing of different fluid elements. For instance the amount of gas in the different temperature regimes, and most importantly in the cold phase, depends sensitively on spatial grid resolution. As an example consider the amount of cold gas, the volume filling factor of which increases for $\sigma / \sigma_{\mathrm{Gal}}=2$ from $\sim 9 \%$ in the $2.5 \mathrm{pc}$ resolution calculations to $\sim 17 \%$ in the $1.25 \mathrm{pc}$ and $0.625 \mathrm{pc}$ resolution cases (see Fig. 7); and the discrepancy for the cold remains still large, when one increases the supernova rate to $\sigma / \sigma_{\mathrm{Gal}}=4$. For the hot phase, on the other hand, there is not much difference in $f_{v \text {, hot }}$ with increasing resolution. The largest variation occurs for the smallest $\mathrm{SN}$ rates with a reduction of at most $20 \%$ compared to the values derived from the $\Delta x=2.5 \mathrm{pc}$ resolution simulations. The resolution increase also affects the cool and warm phases in a similar way with an

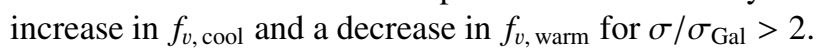

The variations in the volume filling factors are easily understood if one takes into account that with an increase of resolution it becomes possible to resolve the smallest scale structures.
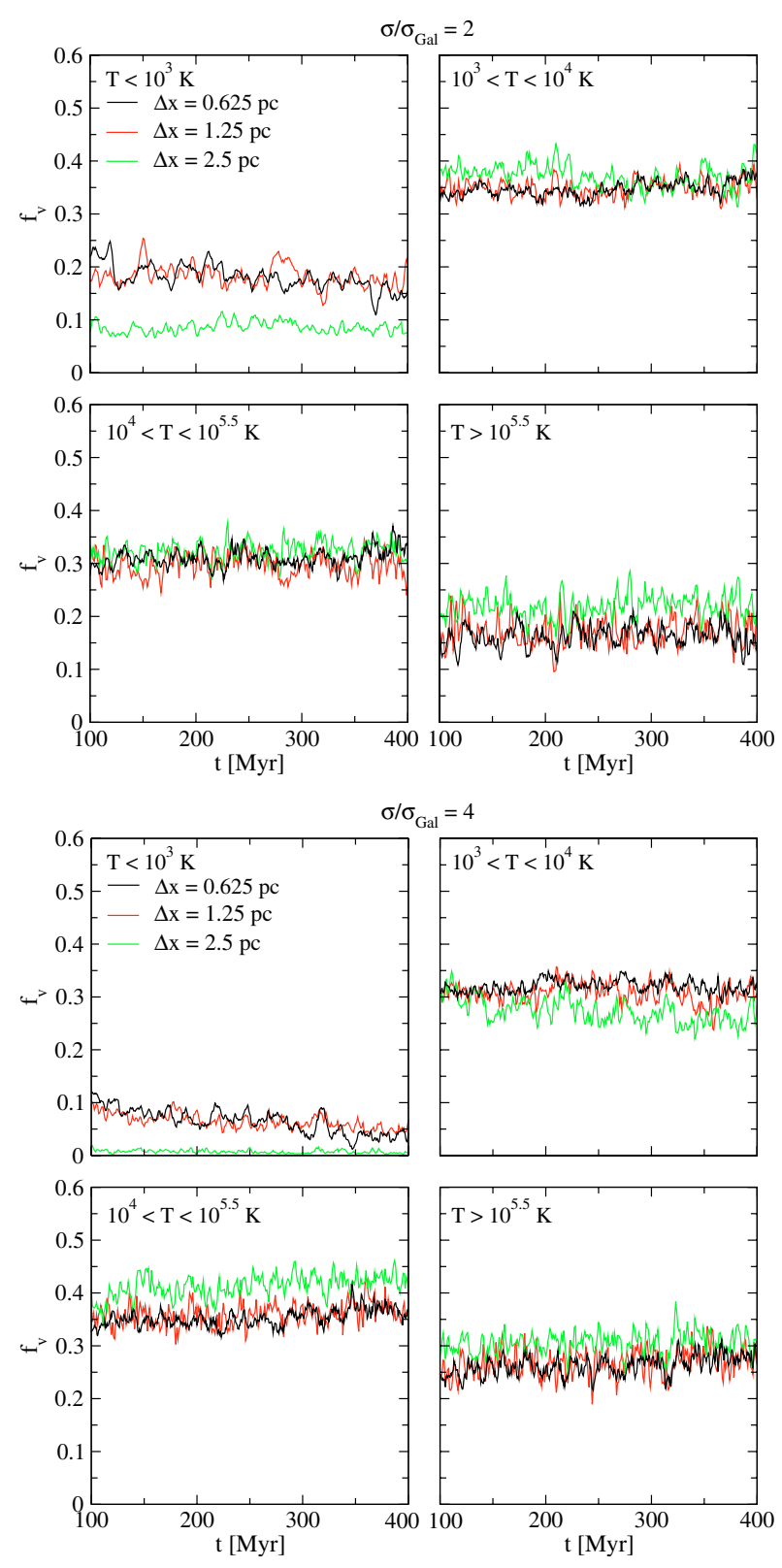

Fig. 7. Comparison between volume filling factors of cold $(T<$ $\left.10^{3} \mathrm{~K}\right)$, cool $\left(10^{3}<T<10^{4} \mathrm{~K}\right)$, warm $\left(10^{4}<T<10^{5.5} \mathrm{~K}\right)$ and hot $\left(T>10^{5.5} \mathrm{~K}\right)$ gas for the finest AMR level grid resolutions of $0.625 \mathrm{pc}$ (black), $1.25 \mathrm{pc}$ (red), and $2.5 \mathrm{pc}$ (green) for the SN rates $\sigma / \sigma_{\mathrm{Gal}}=2($ top panels $)$, and 4 (bottom panels).

Thus instead of averaging out the gas density over larger cells, and thereby wiping out density peaks, radiative cooling as a nonlinear process can become more efficient, since high density regions contribute more to the energy loss rate than low density regions can compensate by an accordingly lower rate. Since cooling is most efficient for dense gas, the cool phase is affected most. In addition, the spatial resolution of shear layers and contact surfaces, gives rise to an increased level of turbulence and a larger number of mixing layers. The latter is most important, because it allows for a faster mixing between parcels of gas with different temperatures (conduction or diffusion processes being of second order and hence inherently slow in nature). The small scale mixing in these simulations is promoted 

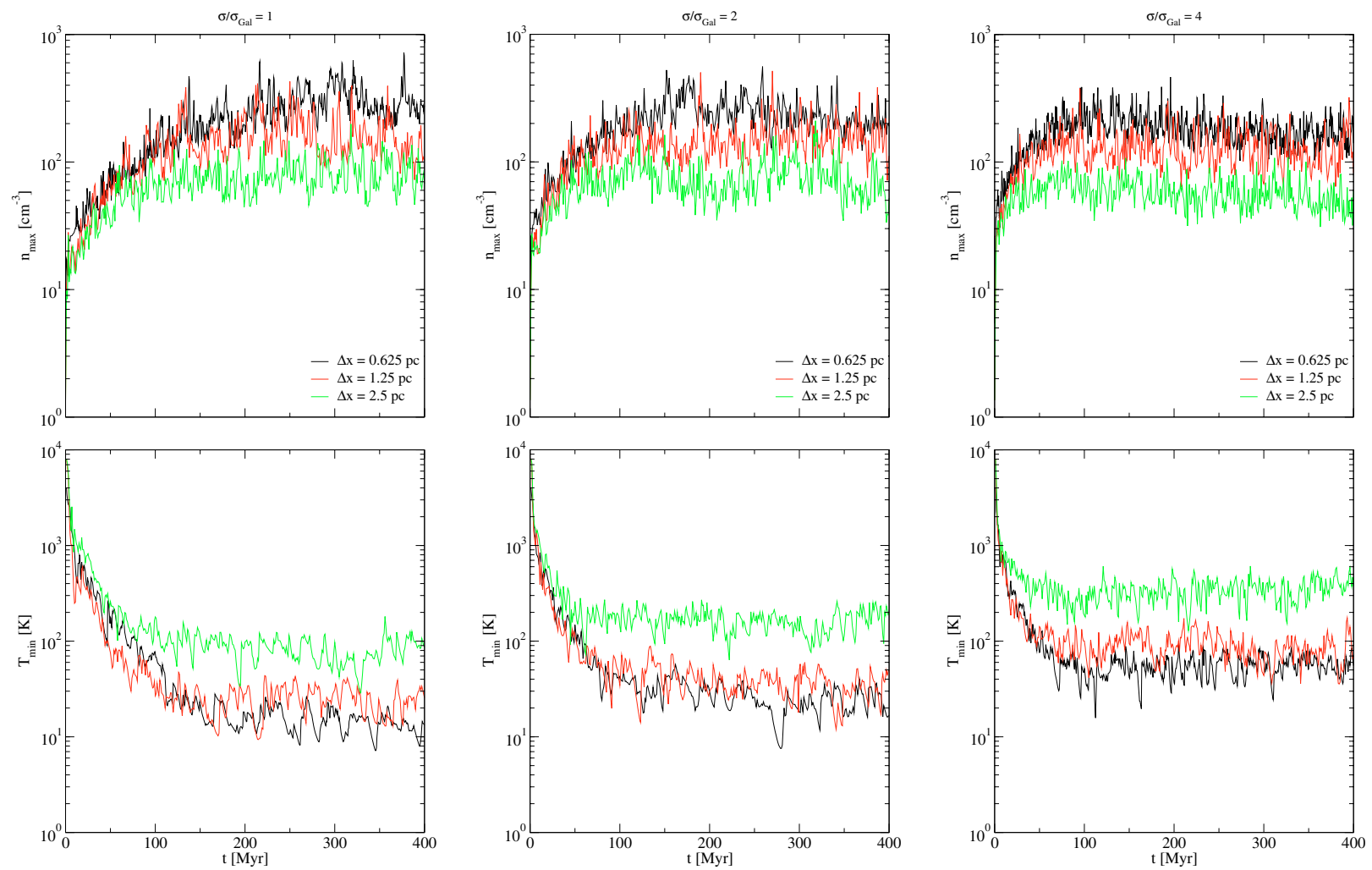

Fig. 8. Comparison between grid resolutions of $0.625 \mathrm{pc}$ (black), $1.25 \mathrm{pc}$ (red) and $2.5 \mathrm{pc}$ (green) of maximum density (top panel) and minimum temperature (bottom panel) for $\sigma / \sigma_{\mathrm{Gal}}=1$ (left column), 2 (middle column), and 4 (right column). Note that in any of the panels there is large difference between the 2.5 and $1.25 \mathrm{pc}$ resolutions, while the differences between $0.625 \mathrm{pc}$ and $1.25 \mathrm{pc}$ resolutions are much smaller. This is an indication of the convergence of the simulations in reproducing the physical processes involved in the dynamics and evolution of the ISM, thus representing a fairly realistic distribution of gas in density-temperature space.

by numerical rather than molecular diffusion, and therefore, the time scales for mixing in the different phases to occur is somewhat smaller (because it happens on larger scales) than those predicted by molecular diffusion theory (e.g., Avillez \& Mac Low 2002). However, as already mentioned, turbulent diffusion, as a consequence of the onset of turbulence due to shear flows, will be most efficient.

A comparison between the maximum density, $n_{\max }$, and minimum temperature, $T_{\min }$, measured at the different finer level resolutions reveals that an increase in resolution from $\Delta x=2.5$ to $1.25 \mathrm{pc}$ implies an average increase in $n_{\max }$ and a decrease in $T_{\min }$ by factors greater than 5 at any $\mathrm{SN}$ rate (cf. Fig. 8). When resolution is increased from 1.25 to $0.625 \mathrm{pc}$ the differences between $n_{\max }$ and $T_{\min }$ for all the $\mathrm{SN}$ rates are small and diminish with the increase of $\mathrm{SN}$ rate. The high $T_{\min }$ for $\sigma / \sigma_{\mathrm{Gal}}=4$ at any resolution is due to the higher cooling time of the gas supply with on average higher temperature. Figure 9 compares the average values of $T_{\min }$ and $n_{\max }$ calculated between 200 and $400 \mathrm{Myr}$ of evolution for the three resolutions (for details, see figure caption).

When a resolution of $0.625 \mathrm{pc}$ is used the average minimum temperature and maximum density, $T_{\min }$ and $n_{\max }$, suffer small variations with respect to those determined at $\Delta x=1.25 \mathrm{pc}$ simulations for all the $\mathrm{SN}$ rates discussed above. This is a clear indication of the convergence of the simulations in reproducing the physical processes involved in the dynamics and evolution of the ISM and that the simulations with $\Delta x \leq 1.25 \mathrm{pc}$ can represent the real ISM. From the fit for $\left\langle T_{\min }\right\rangle=8.35 \exp \left(\frac{\Delta x}{1.106 \mathrm{pc}}\right)$, and $\left\langle n_{\max }\right\rangle=442 \exp \left(-\frac{\Delta x}{1.46 \mathrm{pc}}\right)$, respectively, we can see that there is rapid convergence for $\Delta x \leqq 1.1 \mathrm{pc}$.

\section{Discussion}

The filling factors of some of the phases obtained in this study are similar to those estimated by Spitzer (1990), Ferrière (1995) and Avillez (2000), but seem to be in contradiction with predictions of Ferrière (1998) and are not consistent with Korpi et al. (1999).

The first three authors estimated a volume filling factor of the hot gas in the Galactic disk of $\sim 0.2$, while for the cold and neutral phases Ferrière (1995) and Avillez (2000) arrived to similar values. The largest variation between the volume filling factors in Avillez (2000) and the present paper is by far in the value of $f_{v \text {, cold }}$, as a result of the introduction of background heating due to starlight leading to the formation of thermally stable branches at $10^{3.9}<T<10^{4.2} \mathrm{~K}$ and $T<100 \mathrm{~K}$. Most of the cold gas is located in the unstable branch at temperatures below $10^{3} \mathrm{~K}$ (Avillez \& Breitschwerdt 2004b).

Based on models which follow the expansion and contraction phases of SNRs and SBs, Ferrière (1998) estimated the variation of the volume filling factor of the hot gas with $z$. It is argued that near the solar circle, the volume occupation 

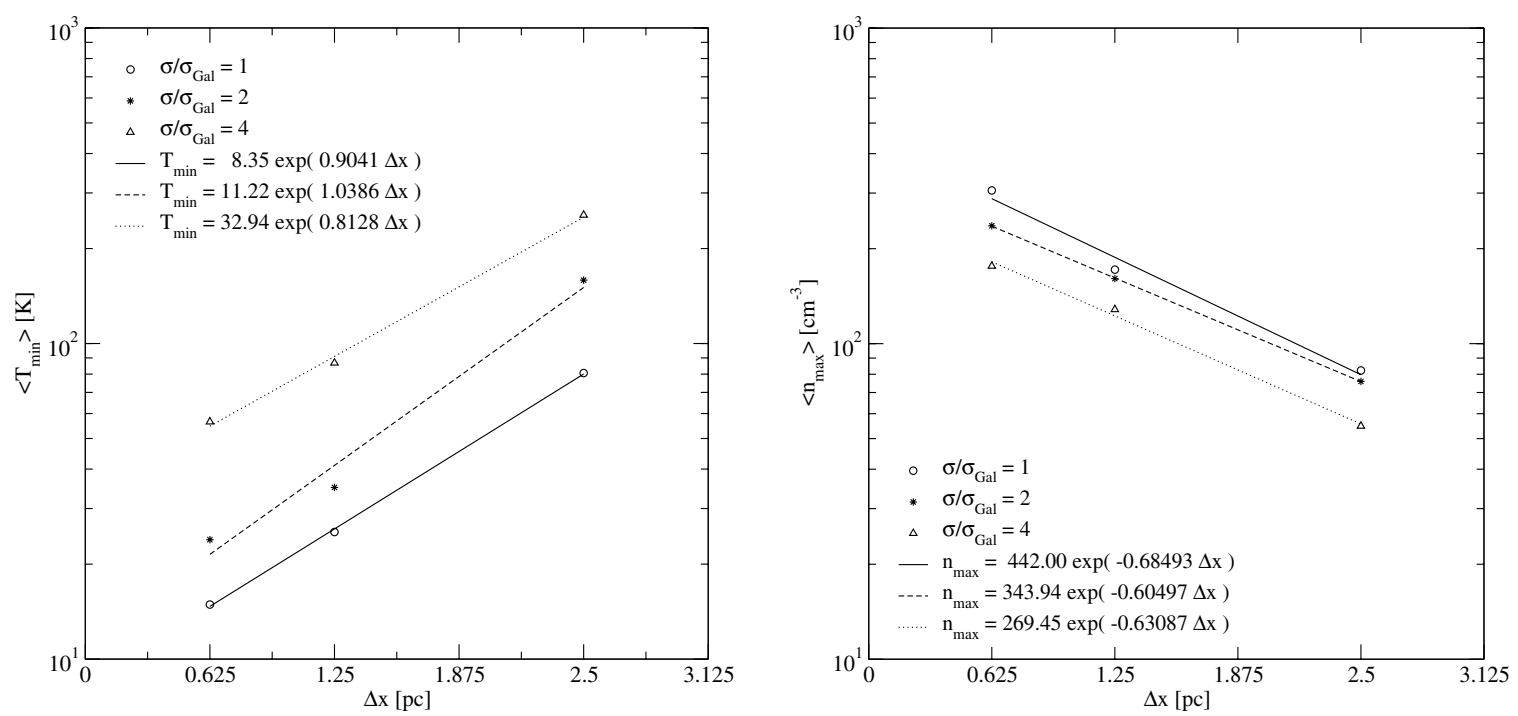

Fig. 9. Comparison between the average minimum temperature (left panel) and maximum density (right panel) as function of resolution for the three SN rates: $\sigma / \sigma_{\mathrm{Gal}}=1$ (open circles), $\sigma / \sigma_{\mathrm{Gal}}=2$ (stars), and $\sigma / \sigma_{\mathrm{Gal}}=4$ (triangles). The plots also show exponential fits to the data points. These average values were calculated during the last $200 \mathrm{Myr}$ of evolution, i.e. after establishing dynamical equilibrium, so that their history does not reflect any memory of the initial conditions.

of the hot gas increases from 0.15 at $z=0 \mathrm{pc}$ to 0.23 at $z=200 \mathrm{pc}$ and decreases gradually for $z>200$ pc (Fig. 12 of Ferrière 1998). The discrepancy between these results and the calculations reported in this paper can be understood by the fact that Ferrière's calculations reflect the distribution of the hot gas inside the SNR and SB cavities whose number decreases with $z$, and therefore the volume filling factor of hot gas decreases accordingly. This contrasts with the present paper where in a global 3D simulation the hot gas is not exclusively connected to individual SNRs or $\mathrm{SBs}^{1}$, but instead can rise into the halo. As the hot gas breaks out of the thick disk, in which its volume filling factor first decreases, it eventually starts filling the entire volume of the Galactic halo. Thus, Ferrière's model does in fact not resolve the vertical gas transport, and hence misses out on the volume fraction of a considerable amount of gas, leading effectively to an increase with $z$-height above $1 \mathrm{kpc}$ as described here.

Figure 2 of Korpi et al. (1999) shows the temperature, density and $P / k$ PDFs for different volumes of the disk: $|z|<0.25$, $|z|<0.5$, and $|z|<1 \mathrm{kpc}$. These are averaged volume-weighted histograms calculated over a period of 50 Myr using six snapshots at equal time intervals between 20 and 70 Myr of evolution. The temperature PDF for the Galactic disk gas has a bimodal distribution with two strong peaks one at $10^{4} \mathrm{~K}$ and another at $\sim 10^{6} \mathrm{~K}$. The latter corresponds to a $f_{v \text {, hot }}$ varying from $20-30 \%$ at $z=0$ pc to $50-60 \%$ for $z=300$ pc. These values are in disagreement with those discussed in the present paper for the Galactic SN rate. The discrepancy is related to the small grid extent in the $z$-direction (with $-1 \leq z \leq 1 \mathrm{kpc}$ ) used by these authors, and consequently the increasing escape of material from the grid with time without any return flow making

\footnotetext{
${ }^{1}$ In fact we see a large number of SNRs and SBs disintegrating on timescales of 1 to $30 \mathrm{Myr}$, respectively, even inside the Galactic disk, where density gradients are on average smaller than in the halo.
}

their model, as the authors argue themselves, only meaningful for a limited length of time, which as we discuss below should not exceed $100 \mathrm{Myr}$.

The need for a duty cycle and the establishment of a global dynamical equilibrium require the use of an extended grid in the direction perpendicular to the Galactic plane. The lack of such an extended $z$-grid inhibits the disk-halo-disk circulation of matter, which otherwise would return gas to the disk sometime later, with noticeably increasing effects for the dynamical evolution as time proceeds, which can be clearly seen in the present simulations. In grids, which have a small vertical extension, e.g., only $1 \mathrm{kpc}$ above and below the midplane ${ }^{2}$, compared to the maximum height to which the hot plasma rises due to the injection of energy and momentum from the sources, most of the gas escapes from the disk in less than $100 \mathrm{Myr}$ (without ever returning to it). Thus the simulations can only be followed for a small evolution time, and therefore, the duty cycle cannot be set up and the system never reaches a dynamical equilibrium state. Moreover, the volume weighted histograms of the thermodynamic properties (e.g., density, pressure and temperature) of the disk gas will retain a memory of the initial evolution. As explained in Sect. 3.1, after an initial collapse of the matter distribution towards the grid midplane, pressure is built up and there is a redistribution of matter on the grid, filling it and allowing a substantial fraction of the gas to escape through the top and bottom boundaries. Therefore, averaged PDFs that including 20-50 Myr snapshots will show the presence of a large fraction of cold gas from the collapse phase (recall the averaged 50 Myr PDF shown in the top panel of Fig. 2 in Sect. 3.2), while PDFs constructed at later times will show the dominance of the hot gas. A combination of these PDFs would give a

\footnotetext{
2 Note, that the maximum extension of the fountain is more than an order of magnitude larger, and therefore such restricted calculations definitely miss an important component of the galactic gas dynamics.
} 
pronounced bimodal distribution (even if there is no background heating due to starlight) corresponding to large volume filling factors for the hot and coolest ISM phases. In other words such PDFs are a reflection of the initial evolution of the simulations and not of the time when a dynamical equilibrium is already established.

\section{Summary and final remarks}

In the present paper we have described a set of 3D hydrodynamical simulations of the interstellar medium in order to study the distribution of the ISM phases and how they vary with increasing star formation rate (i.e., $\mathrm{SN}$ rate) in star forming galaxies. The major goals of this work were (i) to see if the presence of a disk-halo-disk circulation has a major impact on the volume filling factors of the hot phase, and (ii) which minimum grid resolution is needed in order to obtain quantitatively reliable results that can be compared to observations. The main results of the present work can be summarized as follows:

- The occupation fractions of the different ISM phases depend sensitively on the presence of a duty cycle established between the disk and halo working as a pressure release valve for the hot phase.

- The mean occupation fraction of the hot phase varies from about $17 \%$ for the Galactic SN rate to $28 \%$ and $44 \%$ for $\sigma / \sigma_{\text {Gal }}=4$, and 16 , respectively. The mean occupation fraction follows a power law increase with $\mathrm{SN}$ rate, with the power law index of $\sim 0.363$.

- The amount of cold gas picked up in the simulations varies from a value of roughly $19 \%$ (for the Galactic SN rate) to about $5 \%$ for $\sigma / \sigma_{\mathrm{Gal}}=4$ and $\leq 1 \%$ for $\sigma / \sigma_{\mathrm{Gal}} \geq 8$.

- The warm phase has occupation fractions varying between $25 \%$ and $37 \%$ for the three lowest $\mathrm{SN}$ rates $\left(\sigma / \sigma_{\mathrm{Gal}} \leq 4\right)$.

- Background heating is the main reason for the increase in the amount of cold gas in comparison to that in 3D simulations without any background heating.

- A minimum grid resolution of $1.25 \mathrm{pc}$ is needed for quantitatively reliable results, as the convergence towards the $0.625 \mathrm{pc}$ resolution simulations shows.

- A SN rate of 8-16 times the Galactic value already represents a starburst; there is increasing evidence that most star forming galaxies have undergone several such phases during their evolution. In particular for high redshift galaxies, starbursts seem to have been common. Our simulations show that the volume filling factor for the hot phase in the disk increases only moderately from $\left\langle f_{v}\right.$, hot $\rangle=0.35$ and 0.44 for $\sigma / \sigma_{\text {gal }}=8$ and 16 , respectively.

This implies that in X-ray observations, the value of $\left\langle f_{v \text {, hot }}\right\rangle$ in the disk is not a reliable indicator for starburst. Instead the size of the halo in soft X-rays is strongly correlated with a starburst as can be seen from the size of the X-ray halos in recent XMM-Newton observations of NGC 253 (e.g., Pietsch et al. 2001) and NGC 3079 (Breitschwerdt et al. 2004), and also ROSAT observations of M 82 (e.g., Bregman et al. 1995) and NGC 253 (Dahlem et al. 1998; Pietsch et al. 2000).
- Even for Galactic SN rates the fountain cycle is established and thus hot gas is present in galactic halos. This explains why also star forming galaxies with $\mathrm{SN}$ rates comparable to the Galaxy exhibit soft X-ray halos, albeit smaller than in starburst galaxies, as has been observed for NGC 4631 (Wang et al. 2001), which also may have been disturbed by a companion galaxy thereby enhancing the star formation rate, and NGC 891 (Bregman \& Irwin 2002), which is often referred to as the twin galaxy to the Milky Way.

The calculations presented in this paper do not include the magnetic field. A parameter study of the effects of the magnetic field and cosmic rays in the ISM is underway and will be described in Avillez \& Breitschwerdt (2004b). It will deal with the effects of the B-field dissipation, which is too low for Galactic dynamos (Ferrière 1998), as well as with the effects of a weak and strong magnetic field. If the magnetic field is present and is initially mainly oriented parallel to the disk, transport in the halo may be inhibited, although not prevented. On larger scales magnetic tension forces become weaker than on the smallest scales and therefore vertical expansion might still take place efficiently. Either way the occupation fraction of the hot gas could be comparable to the values observed in the present simulations.

Acknowledgements. M.A. and D.B. are partially supported by the ESO/FCT (Portuguese Science foundation) grant PESO/P/PRO/40149/2000. D.B. acknowledges support from the Bundesministerium für Bildung und Forschung (BMBF) by the Deutsches Zentrum für Luft- und Raumfahrt (DLR) under grant 50 OR 0207 and the Max-Planck-Gesellschaft (MPG). The $0.625 \mathrm{pc}$ simulations discussed in this paper were carried out at Compaq Benchmark Center, Toronto, Canada. The authors thank Michael Adamson, team leader at Compaq, for all the support and help during the time of computing.

\section{References}

Avillez, M. A. 2000, MNRAS, 315, 479

Avillez, M. A., \& Berry, D. L. 2001, MNRAS, 328, 708

Avillez, M. A., \& Mac Low, M.-M. 2001, ApJ, 551, L57

Avillez, M. A., \& Mac Low, M.-M. 2002, ApJ, 581, 1047

Avillez, M. A., \& Breitschwerdt, D. 2004a, Baltic Astron., 13, 386

Avillez, M. A., \& Breitschwerdt, D. 2004b, in preparation

Bell, J., Berger, M., Saltzman, J., \& Welcome, M. 1994, SIAM J. Sci. Comp., 15, 127

Berger, M. J., \& Colella, P. 1989, J. Comp. Phys., 82, 64

Breitschwerdt, D., McKenzie, J. F., \& Völk, H. J. 1993, A\&A, 269, 54

Breitschwerdt, D., Pietsch, W., Vogler, A., Read, A. M., \& Trinchieri, G. 2004, in preparation

Bregman, J. N., \& Irwin, J. A. 2002, ApJ, 565, L13

Bregman, J. N., Schulman, E., \& Tomisaka, K. 1995, ApJ, 439, 155

Brinks, E., \& Bajaja, E. 1986, A\&A, 169, 14

Colella, P., \& Woodward, P. 1984, J. Comp. Phys., 54, 174

Cowie, L. L., \& McKee, C. F. 1977, ApJ, 211, 135

Dahlem, M., Weaver, K. A., \& Heckman, T. M. 1998, ApJS, 118, 401

Dalgarno, A., \& McCray, R. A. 1972, ARA\&A, 10, 375

Dettmar, R.-J. 1992, Fund. of Cosm. Phys., 15, 143

Dickey, J. M., \& Lockman, F. J. 1990, ARA\&A, 28, 215

Ferrière, K. M. 1995, ApJ, 441, 281

Ferrière, K. M. 1998, ApJ, 503, 700 
Freeman, K. C. 1987, ARA\&A, 25, 603

Innanen, K. A. 1973, Ap\&SS, 22, 393

Kahn, F. D. 1981, in Investigating the Universe, ed. F. D. Kahn (Dordrecht: Reidel), 1

Korpi, M. J., Brandenburg, A., Shukurov, A., Tuominen, I., \& Nordlund, A. 1999, ApJ, 514, L99

MacLow, M.-M., \& McCray, R. 1988, ApJ, 324, 776

Malyshkin, L., \& Kulsrud, R. 2001, ApJ, 549, 402

McKee, C. F., \& Ostriker, J. P. 1977, ApJ, 218, 148

Mineshige, S., Shibata, K., \& Shapiro, P. R. 1993, ApJ, 409, 663

Miyamoto, M., \& Nagai, R. 1975, PASJ, 27, 533

Narayan, R., \& Medvedev, M. V. 2001, ApJ, 562, L129

Norman, C. A., \& Ikeuchi, S. 1989, ApJ, 345, 372
Pietsch, W., Vogler, A., Klein, U., \& Zinnecker, H. 2000, A\&A, 360, 24

Pietsch, W., Roberts, T. P., Sako, M., et al. 2001, A\&A, 365, L174

Reynolds, R. J. 1987, ApJ, 323, 118

Rosen, A., \& Bregman, J. N. 1995, ApJ, 440, 634

Spitzer, L. 1990, ARA\&A, 28, 71

Strang, W. G. 1968, SIAM J. Numer. Anal., 5, 506

Tomisaka, K. 1998, MNRAS, 298, 797

Wang, Q. D., Immler, S., Walterbos, R., Lauroesch, J. T., \& Breitschwerdt, D. 2001, ApJ, 555, L99

Wolfire, M. G., McKee, C. F., Hollenbach, D., Tielens, A. G. G. M., \& Bakes, E. L. O. 1995, ApJ, 443, 152 